\title{
Atom Probe Tomography: A Technique for Nanoscale Characterization
}

\author{
M. K. Miller* and E. A. Kenik* \\ *Oak Ridge National Laboratory, PO Box 2008, Oak Ridge, TN 37831-6136
}

The atom probe has provided a technique for nanoscale characterization for more than 30 years. The three-dimensional atom probe (3DAP) determines the spatial coordinates and the elemental identities of the atoms within a small volume of a metallic or semiconducting specimen [1]. Fundamentally new analytical techniques have been developed recently to estimate the composition and parameters such as the radius of gyration and number density of features as small as $1 \mathrm{~nm}$ [1]. These techniques, which may be applied to nanoscale features such as embryos, clusters, ultrafine precipitates and the core regions of dislocations, are based on the principle that the solute atoms in a solute-enriched feature are closer together than in the surrounding matrix and permits the atoms associated with these features to be separated from those in the matrix (maximum separation method) [2]. The chemical short range ordering tendencies of solute atoms in each phase and their nearest neighbor configurations may also be statistically estimated. These techniques permit the earliest (pre-precipitation and kinetically controlled) stages of phase separation and decomposition to be investigated. Examples are given for an oxide-dispersion strengthened (ODS) ferritic steel.

Nanoscale clusters and precipitates may be visualized with atom maps in which the position of each solute atom is displayed, Fig. 1a. The solute atoms associated with each cluster may be identified with the maximum separation method described above, Fig. 1b. The size of each cluster may be determined in terms of the radius of gyration or Guinier radius from either the positions of all the solute atoms associated with each cluster or the positions of the atoms of an individual element. Information on the behavior of the different solutes present in the cluster may then be evaluated, Fig. 2. A radial concentration profile with its origin at the center of mass may be constructed for spherical clusters and precipitates to evaluate the tendency for interfacial segregation and to estimate the sharpness of the precipitate-matrix interface, Fig. 3. The composition of individual clusters may be determined by the envelope method: a fine three-dimensional grid is superimposed upon the data; each cluster is defined by all cells of the grid containing the solute atoms identified by the maximum separation method, Fig. 1c; the composition of each cluster is the relative proportions of all atoms within the cells defining the cluster. The outermost cells may be eroded to minimize the influence of cells at the cluster-matrix interface. Alternatively, the composition of a spherical shell with its origin at the center of mass and extending out to specific distances, such as the radius of gyration or the Guinier radius, may be determined. The dependency of the composition on the size of the precipitate may be examined, Fig. 4. The interaction of solutes may also be estimated by determining the composition of the nearest neighbor atoms of shells surrounding a selected atom type [3].

[1] M.K. Miller, Atom Probe Tomography: Analysis at the Atomic Level, Kluwer Academic/ Plenum, New York, 2000.

[2] J.M. Hyde, D. Phil thesis, University of Oxford, Oxford, UK, 1993.

[3] Research at the Oak Ridge National Laboratory SHaRE Collaborative Research Center was sponsored by the Laboratory Directed Research and Development Program and the Division of Materials Sciences and Engineering, U.S. Department of Energy, under contract DE-AC0500OR22725 with UT-Battelle, LLC. 

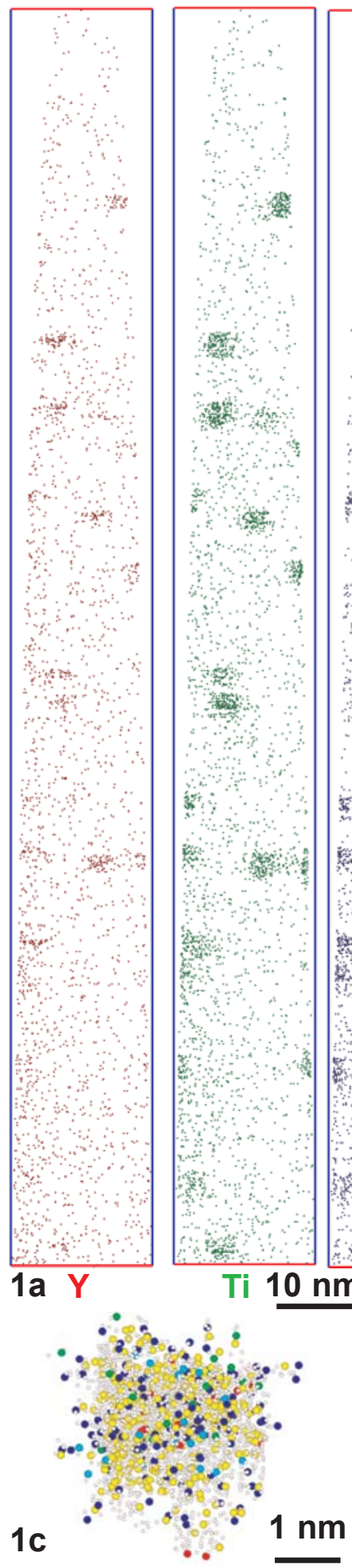
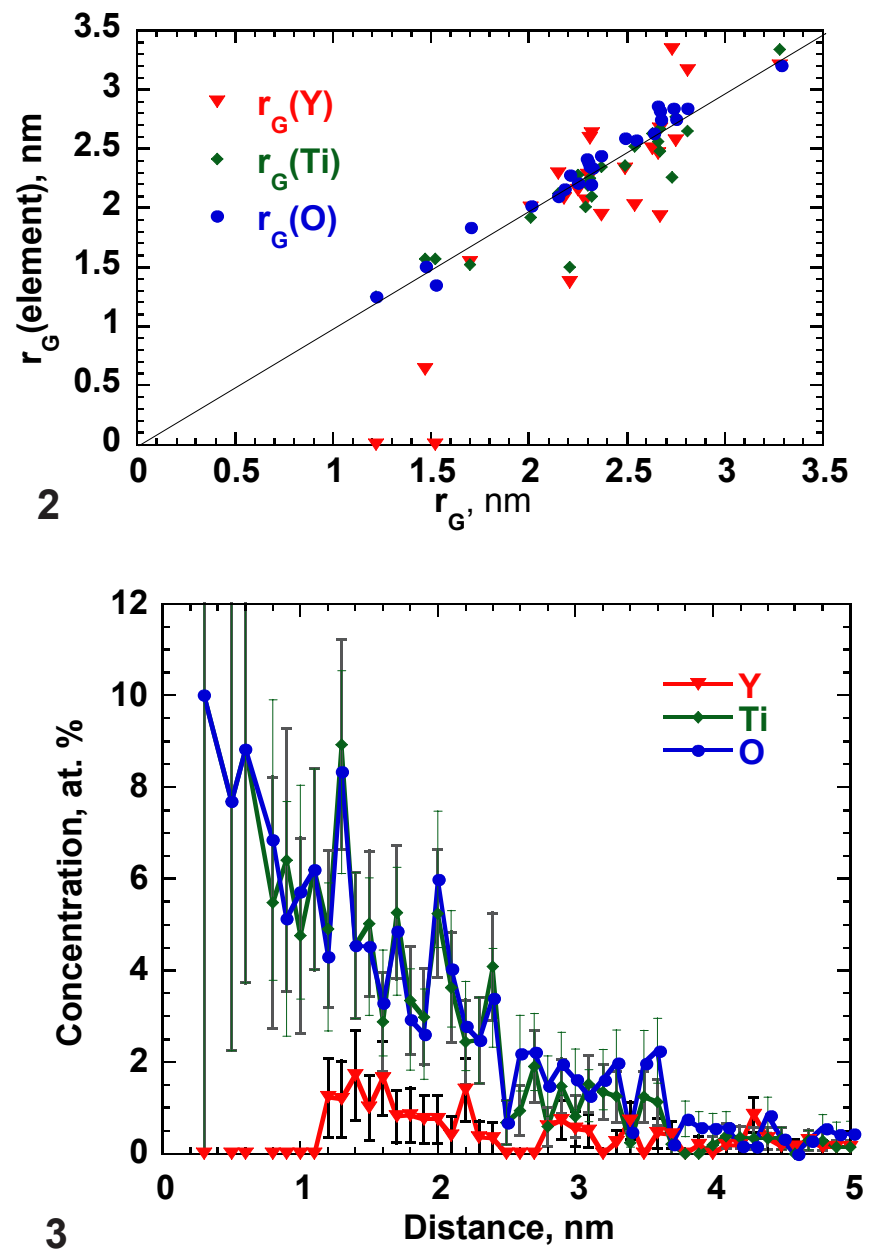

FIG. 1. a) $\mathrm{Y}$, Ti and $\mathrm{O}$ atom maps, b) $\mathrm{Y}$, Ti and $\mathrm{O}$ atoms identified with clusters with the maximum separation method, c) all atoms in a cluster selected by the envelope method.

FIG. 2. Radii of gyration for each element versus the radii of gyration from $\mathrm{Y}+\mathrm{Ti}+\mathrm{O}$ atoms.

FIG. 3. Y, Ti and $\mathrm{O}$ concentrations as a function of distance from the center of mass of a cluster.

FIG. 4. Cluster compositions as a function of the cluster size. 